tions on the displacements about a circular opening in an elastic medium. Gumbel has extended this by outlining one way in which the interaction analysis between an elastic soil and an elastic tunnel liner can be made. By making use of the solutions given in the Note, Gumbel has arrived at the factors required to convert solutions for the soil-liner interaction for load case 1 to those for load case 2 .

With regard to the behaviour of buried pipes and culverts, Fig. 3 of the Note was drawn with that situation in mind. However, recourse to the literature shows that an analysis which is based on the idcalization of the pipe or culvert being surrounded by a uniform elastic material may not be relevant. Höeg (1968) reviews a number of cases in which the measured pressure distributions do not have the symmetry about the horizontal diameter predicted by the simple elastic analysis. He explains that the problem is related to the method of placing the backfill around the pipe. The densities beneath the springline tend to be lower than those above, so that the stiffness of the material surrounding the bottom half of the pipe is less than that over the top half. Höeg (1968) and Valsangkar \& Britto (1979) found that when special placement techniques which gave a uniform backfill were employed, the simple elastic analysis gives a good description of the observed behaviour. However, these idealized placement techniques are not relevant to the field situation. Also in many field cases there are additional complications due to the cutting of a trench for the pipe or the presence of a stiffer material beneath the bottom of the pipe. Thus, the assumption of a uniform elastic surrounding material is even less likely to be true for the pipe or culvert situation that it is for the tunnel case.

\section{REFERENCES}

Höeg, K. (1968). Stresses against underground structural cylinders. J. Soil Mech. Fdns Div., Am. Soc. Civ. Engrs 94, SM4, 833-858.

Valsangkar, A. J. \& Britto, A. M.(1979). Centrifuge tests of flexible circular pipes subjected to surface loading. Supplementary report 520. Crowthorne: Transport and Road Research Laboratory.

\title{
Reinforced earth structures situated on soft foundations
}

\author{
C. J. F. P. JONES and L. W. EDWARDS (1980). Géotechnique 30, No. 2, 207-213
}

\section{D. Bolton, Cambridge University}

The Authors discuss the role of the surrounding soil in the design of a reinforced earth retaining wall. They draw attention to a paradox, namely that reinforced earth walls have proved to be successful when founded on soft soils whereas it is possible to demonstrate that a conventional reinforced concrete retaining wall theoretically mobilizes less damaging base stresses than its more novel counterpart. This is no paradox. The forward projection of a reinforced concrete base beyond the face of a conventional wall obviously has the beneficial effect of displacing the centroid of that base in the direction required to resist the applied overturning moment. Almost any textbook will advise such an offset to counter moments due to dead loads on foundations. If a designer of reinforced earth structures wished to use this technique he could do so by constructing a projecting base platform in reinforced concrete, or even in reinforced earth. This issue has little to do with the selection of a wall type to stand on poor soils. The particular merit of most reinforced earth systems is that the bold joints between the facing panels invest the structure with an almost unrivalled capacity to deform without this being noticed. This leads to the satisfactory use of a reinforced wall with little or no expenditure on foundations, in conditions which would demand the use of piles if a conventional smooth-faced monolith were required.

The Authors go on to claim that 'the global failure criterion is not one of overturning about the toe, as the application of some design methods suggest, but that of a rotational slip through the retained embankment'. In this argument they seemingly confuse the concept of physical rotation with that of the moment of a force. Their contribution evidently seeks to cast doubt by innuendo on the method of enhancing the vertical basc strcss supposed to act under the toe of a reinforced earth wall by a factor $1+k_{\mathrm{a}} \mathrm{H}^{2} / \mathrm{L}^{2}$ due to over-turning stresses $k_{\mathrm{a}} \gamma \boldsymbol{Z}$ which are taken to act horizontally on the reinforced zone. This calculation is, of course, a manifestation of engineers' beam theory, and invites the designer of a retaining wall to consider the foundation soils on which it stands as equivalent to the cross-section of a wide column. If the wall has to carry a moment of $k_{\mathrm{a}} \gamma H^{3} / 6$ from its backfill, then it must create a counterbalancing reaction at its base. On the further assumption that the induced-stress distribution is linear, the result is an increment of $k_{\mathrm{a}} \gamma H^{3} / L^{2}$ to add to the overburden pressure $\gamma H$ at 
the toe. Some corollaries include the well-known requirement that the shear force, in this case equal to $k_{\mathrm{a}} \gamma \mathrm{H}^{2} / 2$, is parabolically distributed. Of course, such assumptions cannot represent the case in hand exactly. They are crude and simple and should therefore be challenged when it can be demonstrated that an improvement is possible.

The Author's main weapon in their attack on this simple stress-enhancement concept is their observation that in certain cases walls may rotate inwards. It is surprising that they think that this is strange, and more surprising that they see it as evidence against stress enhancement at the toe. Is there a paradox in 'outward' moments coinciding with 'inward' rotations? Consider an elastic footing carrying a uniformly distributed pressure and resting on a homogeneous bed of elastic soil. Sagging, or 'outward' bending moments will clearly exist as the foundation dishes. Also, the soil contact stresses will be greatest around the edge of the footing, correlating with the fact that the evident dishing will be less than if the footing had been perfectly flexible. Consequently there is stress enhancement at an edge of a foundation which suffers an inward rotation: there is no paradox here. If the Authors are to establish a better design method they should address themselves to stresses in addition to strains. The two key assumptions are that the lateral earth pressures applied by the backfill are 'active' justifying the use of $k_{\mathrm{a}}=\left(1-\sin \phi^{\prime}\right) /\left(1+\sin \phi^{\prime}\right)$, and that they are horizontal. Viewed in isolation, the inward rotations invoked by the Authors may lead to the opinion that passive earth pressures may be generated, which would lead to an even greater toe stress: this is evidently not what they had in mind. If friction were to be invoked between the backfill and the reinforced zone then the first question must be 'In which direction? If the backfill settled more than the reinforced zone the friction would be downwards on that zone; this would increase the average base stress but reduce the overall 'outward' or 'overturning' moment. If the designer took advantage of this he might later regret any ground displacements which tended to reverse the effect; possibilities are a lens of more compressible soil beneath the reinforced zone, a lens of less compressible soil beneath the backfill, drainage, subsidence following the approach of long-wall mining of some bedrock, or the reinstatement of a deep service trench behind the reinforced zone.

If their contribution is to be read as an invitation to consider in some detail the possible limit states in to which a reinforced earth retaining wall should not be allowed to pass, then it is to be hoped that the Authors' challenge will be taken up. Such challengers should be careful to distinguish between collapse limit states and the approximate procedures such as the stress-enhancement concept which are necessary to add substance to them, and serviceability limit states which may demand predictions of deformations for clients with tight dimensional constraints. If such a revision is to be useful, it should also make clear under what conditions the limit states will actually be avoided. What conditions should be imposed on the site investigation for retaining walls if the designer is safely to mobilize supportive friction against the backfill? Of what magnitude is that friction to be? What conditions must be placed on future earthworks in that vicinity? In particular, are global savings to be expected from this new element of optimism in the designer's calculations? These questions remain to be answered.

\section{Authors' reply}

There is no disagreement with $\mathrm{Mr}$ Bolton's analysis of the merits of reinforced earth as a structure to be preferred on poor soils, but it would appear that his contribution is based upon a misunderstanding of the basic argument in the Technical Note which relates to the difficulties facing some designers of reinforced earth structures.

At present, many designs are prepared in accordance with published design memoranda and it is the interpretation of these memoranda which can lead to problems for the engineer. As an example, the Department of Transport Memorandum BE 3/78 can lead the designer to the situation in which the bearing pressures beneath the base of the structure become the limiting criteria for design and therefore of structural form. If bearing pressure is the limiting criterion, then Fig. 1 of the Technical Note clearly shows that a conventional cantilever structure is to be preferred to a reinforced earth structure. Further, the trapezoidal pressure distribution (Fig. 1) is referred to in the Memorandum under the section considering 'tilt' and the underlying assumption is that the structure is tilting forward with an anticlockwise rotation. The practical outcome is that the designer consciously seeking to produce a reinforced earth solution, chosen because of its known advantages when weak sub-soils are encountered, finds that the Design Memorandum frustrates his efforts; hence the paradox.

The Technical Note seeks to illustrate this weakness and also to show that the assumption concerning rotations and tilts can be in error. The overall behaviour mechanism of reinforced earth depends upon the nature of the sub-soil, not upon the theoretical trapezoidal bearing distribution. Thus a structure founded upon a stiff sub-soil may tilt forward in a clockwise direction, while the behaviour of reinforced earth walls on a soft 
foundation may rotate in a positive direction (Figs 2 and 3).

Rather than rely upon a criterion based upon bearing-pressure distribution the Technical Note shows that stability analysis of the overall reinforced earth problem is possible using readily available non-linear finite element techniques. Use of this technique also demonstrates that the overall behaviour and stability of a reinforced earth structure is a function of the geometry of the design problem-a point not covered in the published Design Memorandum. 